\title{
Frutanos tipo inulina associados à dieta de cafeteria: efeito no perfil cardiometabólico de ratos Wistar
}

\section{Inulin-type fructan associated with cafeteria diet: effect on the cardiometabolic profile of Wistar rats}

\author{
Francielle Cássia Silva ${ }^{1}$, Maria Aparecida de Lima Oliveira, Lívia Bruni de Souza, \\ Thaiany Goulart de Souza e Silva², Hudsara Aparecida de Almeida Paula ${ }^{3}$, Débora \\ Vasconcelos Bastos Marques ${ }^{4}$
}

\section{Resumo}

\begin{abstract}
A dieta de cafeteria foi desenvolvida a fim de mimetizar a dieta ocidentalizada, uma vez que esta associa-se a desordens no perfil cardiovascular, como dislipidemias, hiperglicemia, acúmulo de gordura corporal, entre outros. Os frutanos tipo inulina (FTI) são fibras solúveis categorizados como prebióticos. O presente estudo objetivou avaliar os efeitos da dieta de cafeteria associadas aos FTI sobre o perfil cardiometabólico em ratos Wistar. Para tanto, utilizou-se 30 animais. Os FTI purificados, bem como, provenientes da matriz alimentar (yacon em pó), foram adicionados a seus respectivos grupos experimentais na dose de $6 \%$, durante 30 dias. As variáveis analisadas foram: composição centesimal e conteúdo de FTI, ganho de peso (GP), peso relativo do ceco (PRC), peso relativo do fígado (PRF), consumo alimentar, glicemia, triglicerídeos, colesterol total, HDL-c, LDL-c, tecido adiposo subcutâneo e visceral (epididimal, retroperitoneal e mesentérica). Todas as análises seguiram os protocolos padronizados. A análise estatística foi realizada no software Sigma Stat 4.0 e teste de normalidade de Shapiro-Wilk, testes descritivos, ANOVA ou teste de Kruskal-Wallis seguidos dos testes de comparação de médias de Duncan ou Dunn's. O nível de significância adotado foi de $p<0,05$. A variável PRC foi estatisticamente maior nos grupos submetidos aos FTI. O grupo que recebeu dieta de cafeteria apresentou valores superiores no GP, VLDL, triglicerídeos, tecido adiposo subcutâneo e epididimal. Não houve diferença estatística nos parâmetros PRF, colesterol total, LDL-c, HDL-c e glicemia. Portanto, através deste trabalho, verificou-se efeitos sistêmicos benéficos dos FTI em parâmetros como: PRC, GP, VLDL, triglicerídeos, tecido adiposo.
\end{abstract}

Palavras chave: Frutanos. Inulina. Cardiovascular. Dieta Ocidental.

\begin{abstract}
The cafeteria diet was developed in order to mimic the western diet, since it is associated with disorders in the cardiovascular profile, such as dyslipidemia, hyperglycemia, accumulation of body fat, among others. Inulin-type fructan (ITF) are soluble fibers categorized as prebiotics. The present study aimed to evaluate the effects of ITF associated with cafeteria diet on the cardiometabolic profile in Wistar rats. For this, 30 animals were used. The purified ITFs, as well as from the food matrix (yacon powder),

1 Graduanda em Nutrição pela Universidade Federal de Alfenas, Alfenas, Minas Gerais, Brasil. E-mail: franciellec.silva@hotmail.com

Nutricionista pela Universidade Federal de Alfenas, Alfenas, Minas Gerais, Brasil.

3 Doutorado em Ciência e Tecnologia de Alimentos pela Universidade Federal de Viçosa, Viçosa, Minas Gerais, Brasil. Docente Adjunta da Universidade Federal de Alfenas, Alfenas, Minas Gerais, Brasil Doutorado em Ciência e Tecnologia de Alimentos pela Universidade Federal de Viçosa, Viçosa, Minas Gerais, Brasil.

4 Doutoranda em Biociências aplicadas à Saúde pela Universidade Federal de Alfenas, Alfenas, Minas Gerais, Brasil.
\end{abstract}


were added to their respective experimental groups at a dose of $6 \%$ for 30 days. The analyzed variables were: centesimal composition and ITF content, weight gain (WG), relative weight of cecum (RWC), relative liver weight (RLW), dietary intake, glycemia, triglycerides, total cholesterol, HDL-c, LDL -c, subcutaneous and visceral adipose tissue (epididimal, retroperitoneal and mesenteric). All analyzes followed the standardized protocols. Statistical analysis was performed using the Sigma Stat 4.0 software and a Shapiro-Wilk normality test, descriptive tests, ANOVA or Kruskal-Wallis test followed by the Duncan or Dunn's averages comparison tests were performed. The level of significance was set at $\mathrm{p}<0.05$. The RWC variable was statistically higher in the groups submitted to FTI. The group that received a cafeteria diet presented higher values in WG, VLDL, triglycerides, subcutaneous and epididimal adipose tissue. There was no statistical difference in the parameters RLW, total cholesterol, LDL-c, HDL-c and glycemia. Therefore, through this work, beneficial systemic effects of ITF were observed in parameters such as: RWC, WG, VLDL, triglycerides and adipose tissue.

Keywords: Fructans. Inulin. Cardiovascular. Western Diet.

\section{Introdução}

As doenças cardiometabólicas estão entre umas das principais causas de mortalidade em humanos. Acredita-se que a microbiota intestinal esteja relacionada com anormalidades cardiometabólicas e hábitos alimentares. ${ }^{(1)}$ Além da dieta, fatores fisiológicos e ambientais, como estilo de vida e utilização de antibióticos, tornam o ambiente intestinal particular entre os indivíduos. ${ }^{(2)}$

O conteúdo excessivo de gordura na alimentação contribui para diversas doenças como obesidade, diabetes e doenças cardiovasculares. ${ }^{(3)}$ Sendo assim, com o crescente consumo de alimentos de elevada densidade energética e palatibilidade desenvolveuse a dieta de cafeteria para animais de laboratório com o objetivo de simular a realidade humana, além de promover aumento do peso corporal, circunferência da cintura, hiperinsulinemia, hiperglicemia e esteatose hepática. ${ }^{(4)}$

Diante desta realidade, a comunidade científica, nos últimos anos, tem explorado os benefícios dos alimentos funcionais, ou seja, ingredientes que quando consumidos exercem funções além da nutrição, com atuação em processos fisiológicos, assim como, na redução de enfermidades e promoção da qualidade de vida. ${ }^{(5)}$ Dentre os alimentos funcionais destacam-se os prebióticos, probióticos e simbióticos. ${ }^{(6)}$

Os prebióticos não são hidrolisados pelo trato gastrointestinal, sendo utilizados como substrato para bactérias no cólon, promovendo, desta forma, alterações na microbiota e efeitos sistêmicos para a saúde do consumidor. ${ }^{(7)}$

$\mathrm{Na}$ classe dos prebióticos estão inseridos os frutanos, como a inulina e frutooligossacarídeos (FOS). Trata-se de substâncias com propriedade funcionais e entidades químicas similares, ou seja, ambas apresentam ligações $\beta(2 \rightarrow 1)$ de unidades de frutose terminadas em uma unidade de glicose. Porém, o número de unidades de monossacarídeos que compõe a molécula, denominado de grau de polimerização, permite a diferença entre estes compostos. ${ }^{(8)}$ Recomenda-se ingerir 4 a 5 gramas de frutanos na alimentação diária. Estes são amplamente encontrados na alimentação, sendo obtido por meio do alho, alcachofra, banana, cebola, chicória, tomate, cevada, aspargos, entre outros. ${ }^{(9)}$ Para Javier Fernández e colaboradores, ${ }^{(10)}$ FOS e inulina, são substâncias promissoras, inclusive, quando associadas a dieta ocidental.

Além destes, a batata Yacon é exemplo de alimento fonte de frutanos, FOS e inulina. ${ }^{(11)}$ Segundo Caetano ${ }^{(12)}$ este alimento apresenta de $6,4 \%$ a $70 \%$ de FOS do peso e relaciona-se com redução na glicemia, peso corporal, homeostase lipídica, bem como, câncer colorretal em humanos.

Sendo assim, o presente estudo tem como objetivo avaliar os efeitos da dieta de cafeteria associados com frutanos tipo inulina sobre o perfil cardiometabólico em ratos Wistar. 


\section{Material e Métodos}

Aquisição do Yacon em pó, FOS e Inulina purificados

O yacon em pó, industrializado, foi adquirido no mercado local do município de Alfenas-MG. O FOS e a inulina, ambos purificados, da marca Eficácia ${ }^{\circledR}$ foram adquiridos no município de Belo Horizonte-MG.

Composição química e teor de frutanos tipo inulina do yacon em pó

As análises de composição centesimal do yacon em pó (YP) foram realizadas de acordo com protocolos oficiais: umidade, determinada em estufa a $105^{\circ} \mathrm{C}$ até se atingir o peso estável; proteínas, pelo método de Kjeldahl, de modo que o teor proteico calculado pela multiplicação do teor de nitrogênio pelo fator 6,25; lipídeos, pela técnica de extração com éter etílico em equipamento Soxhlet; e cinzas, por processo gravimétrico, a partir da carbonização das amostras, prosseguida de incineração em mufla a $550{ }^{\circ} \mathrm{C}$ até peso constante. ${ }^{(13)}$

Para determinar as concentrações de FOS e inulina, as amostras foram analisadas pelo Centro de Raízes e Amidos Tropicais (CERAT), Botucatu-SP.

As concentrações dos carboidratos não digeríveis (FOS e inulina) do yacon em pó, foram determinadas através de cromatografia líquida de alta eficiência (HPLC), de acordo com os métodos de Kaneko, Kudo e Horikoshi, ${ }^{(14)}$ com uma coluna BIO-RAD HPX 87P (fase estacionária de chumbo), utilizando água purificada como fase móvel. As amostras foram diluídas (1g em 100mL de água destilada), centrifugadas a $11.269 \mathrm{x} \mathrm{g}$, e, logo adiante, foram filtradas por meio de uma membrana de decafluoreto (PVDF) da polivinil Millipore com 0,22 um de porosidade e $13 \mathrm{~mm}$ de diâmetro. A cromatografia em que as amostras foram submetidas é de alta eficiência (HPLC) (Varian, ProStar 410 HPLC Auto Sampler, Varian Inc, USA) com detector de índice de refração e injetor automático, em $0,6 \mathrm{~mL}$ min.1 de taxa de fluxo e $85^{\circ} \mathrm{C}$ de temperatura de coluna.

\section{Ensaios biológicos}

Aspectos éticos

O presente estudo foi aprovado pela Comissão de Ética para Uso de Animais, da Universidade Federal de Alfenas/UNIFAL, registro 037/2016. Todos os procedimentos adotados estão conforme o preconizado pelo Colégio Brasileiro de Experimentação Animal.

\section{Animais experimentais}

Foram utilizados 30 ratos (Rattus norvegicus, variedade albinus, classe Rodentia), da raça Wistar, recém-desmamados (21 dias de idade) provenientes do Biotério Central da Universidade Federal de Alfenas. Os animais foram mantidos em gaiolas individuais de aço inoxidável, em ambiente de temperatura $\left(22 \pm 2^{\circ} \mathrm{C}\right)$ e luz controladas, em fotoperíodo de 12 horas e receberam água potável ad libitum.

\section{Delineamento experimental}

$\mathrm{O}$ experimento foi conduzido durante o período de 30 dias. O estudo foi realizado por meio de Delineamento Inteiramente Casualizado (DIC). Os animais foram alocados em 5 grupos $(n=6)$ : Cafeteria $(\mathrm{CAF})$, Cafeteria + Yacon em pó $(\mathrm{CAF}+\mathrm{YP})$, Cafeteria + FOS (CAF+FOS), Cafeteria + inulina $(\mathrm{CAF}+\mathrm{I})$, Cafeteria + FOS e inulina $(\mathrm{CAF}+\mathrm{FI})$.

\section{Dietas experimentais}

Um grupo de animais recebeu a dieta de cafeteria, outro recebeu a dieta de cafeteria acrescida de yacon em pó, outro grupo recebeu a dieta de cafeteria acrescida de FOS purificada, outro grupo foi tratado com dieta de cafeteria adicionada de inulina purificada e o último grupo foi tratado com FOS e inulina purificados. O grupo FOS e o grupo Inulina, receberam $6 \%$ de cada um dos prebióticos e o grupo 
FOS e inulina recebeu uma quantidade de $3 \%$ de FOS e $3 \%$ de inulina. A quantidade média de dieta administrada foi de $18 \mathrm{~g}$ diários variando-se a oferta entre 15 a 20 g diários, ${ }^{(15)}$ o que era controlado através do cálculo de restos e sobras. Após o preparo das rações, estas foram acondicionadas hermeticamente em sacos de polietileno, identificados e refrigerados
(10-15 $\left.{ }^{\circ} \mathrm{C}\right)$ até o momento da sua utilização. Para fornecer a dieta aos animais, pesava-se e anotava a quantidade diária, para em seguida, fazer a distribuição da mesma, ainda congelada.

A Tabela 1 descreve a composição da dieta de cafeteria ${ }^{(16,15)}$ adaptada com a especificação quantitativa dos frutanos.

Tabela 1 - Composição (g/100g) das dietas dos grupos experimentais (Alfenas, 2017).

\begin{tabular}{cccccc}
\hline Componentes & \multicolumn{5}{c}{ Grupos experimentais } \\
\hline & CAF & CAF + YP & CAF + FOS & CAF + I & CAF + FI \\
Biscoito doce & 14,28 & 14,28 & 14,28 & 14,28 & 14,28 \\
Batata Palha & 14,28 & 14,28 & 14,28 & 14,28 & 14,28 \\
Chocolate ao leite & 14,28 & 14,28 & 14,28 & 14,28 & 14,28 \\
Bacon & 14,28 & 14,28 & 14,28 & 14,28 & 14,28 \\
Patê de sardinha & 28,57 & 28,57 & 28,57 & 28,57 & 28,57 \\
Dieta comercial & 14,28 & 8,28 & 8,28 & 8,28 & 8,28 \\
Yacon em pó & - & 6,00 & - & - & - \\
FOS purificada & - & - & 6,00 & - & 3,00 \\
Inulina purificada & - & - & - & 6,00 & 3,00 \\
& & Total: 100,00 & &
\end{tabular}

Fonte: Autores.

CAF: Grupo controle com dieta de cafeteria; CAF+YP: Grupo dieta de cafeteria suplementada com $6 \%$ de FOS e Inulina do Yacon em pó; CAF+FOS: Grupo dieta de cafeteria suplementada com $6 \%$ de FOS purificada; CAF+I: Grupo dieta de cafeteria suplementada com 6\% de inulina purificada; CAF+FI: Grupo dieta de cafeteria suplementada com 3\% de FOS e 3\% de inulina purificados.

Para determinar a composição da dieta de cafeteria, as amostras foram analisadas pelo Centro de Raízes e Amidos Tropicais (CERAT), Botucatu-SP. Utilizou-se a metodologia da AOAC (Association of Official Analytical Chemists). ${ }^{(13)}$

\section{Eutanásia dos animais}

Para proceder a eutanásia, os animais foram pesados e anestesiados com cloridrato de quetamina $(0,1 \mathrm{mg} / \mathrm{kg}$ peso corporal $)$ e cloridrato de xilazina $(0,05 \mathrm{mg} / \mathrm{kg}$ peso corporal) e posteriormente foi feita uma incisão na região abdominal para serem sacrificados por perfusão cardíaca sob anestesia. Foram retiradas amostras de sangue para as dosagens bioquímicas mencionadas. Após a morte do animal, foi retirado o ceco, o qual foi lavado com solução fisiológica antes de proceder à pesagem do órgão.

\section{Análises bioquímicas}

No final do experimento, após jejum de 6 horas, foi coletado o sangue por punção cardíaca para a determinação de glicemia, triglicerídeos, colesterol total e HDL-colesterol. As análises foram realizadas utilizando o Kit Analisa ${ }^{\circledR}$, e a metodologia de acordo com instruções do fabricante. Após as análises bioquímicas, os resíduos biológicos e reagentes foram descartados separadamente e encaminhados pela Universidade a empresas especializadas no descarte. 
A lipoproteína de baixa densidade (LDLcolesterol) foi calculada segundo a fórmula de Friedewald, Levy e Frederickson ${ }^{(17)}$ de forma que $\mathrm{LDL}=\mathrm{CL}-\mathrm{HDL}-(\mathrm{TG} / 5)$, onde CL refere-se a colesterol, HDL significa High Density Lipoproteins (lipoproténas de alta densidade), e, TG se refere a triglicerídeos. $^{(18)}$ A Lipoproteína de densidade muito baixa (VLDL) foi calculada da seguinte forma: VLDL=Triglicerídeos $/ 5$.

Análise do peso relativo do ceco e do peso relativo do fígado

Para a obtenção dos pesos, foi utilizada a balança analítica $\left(\mathrm{Gehaka}^{\circledR}\right)$. O peso corporal dos animais era aferido semanalmente, para tanto, os animais eram colocados em recipiente (com peso deste descontado). A determinação do peso relativo do ceco se deu conforme a equação: [peso relativo do ceco $=$ peso do ceco $(\mathrm{g}) \div$ peso corporal final (g)]. Para o peso relativo do fígado foi adotado o mesmo raciocínio. O peso relativo é mais fidedigno quando comparado com o peso absoluto, por levar em consideração o peso corporal também.

\section{Análise estatística}

As análises estatísticas foram realizadas no software Sigma Stat 4.0 e foram utilizados o teste de normalidade de Shapiro-Wilk, testes descritivos, ANOVA ou teste de Kruskal-Wallis seguidos dos testes de comparação de médias de Duncan ou Dunn's. O nível de significância adotado foi de $\mathrm{p}<0,05$.

\section{Resultados}

A análise centesimal do yacon em pó e da dieta de cafeteria são apresentadas na Tabela 2.

Tabela 2 - Composição química do yacon em pó e dieta de cafeteria g/100g (Botucatu, 2017).

\begin{tabular}{ccc}
\hline Análises & Yacon em pó (YP) & Dieta de Cafeteria (CAF) \\
\hline Umidade (\%) & 5,63 & 19,10 \\
Proteínas (\%) (kca & 4,16 & 11,11 \\
Lipídeos (\%) & 0,2 & 27,74 \\
Carboidratos (\%) & 86,24 & 26,32 \\
Cinzas (\%) & 3,77 & 4,38 \\
Fibras (\%) & - & 11,35 \\
Calorias (kcal) & 363,4 & 444,78 \\
\hline
\end{tabular}

Fonte: CERAT

A quantidade de frutanos tipo inulina contidas no Yacon em pó está apresentada na tabela 3.

Tabela 3 - Composição de frutanos tipo inulina do yacon em pó (Botucatu, 2017).

\begin{tabular}{cc}
\hline Componente & $\mathbf{g} / \mathbf{1 0 0 g}$ \\
\hline FOS & 24,46 \\
Inulina & 20,65 \\
Frutanos tipo inulina & 45,11 \\
\hline
\end{tabular}

Fonte: CERAT 
A variável Peso Relativo do Ceco (PRC) teve diferença significativa entre os grupos experimentais ( $<<0,001$ ), sendo que o grupo com suplementação de $6 \%$ de FOS purificado, apresentou maior PRC $(0,614 \pm 0,145)$. Já o grupo que recebeu apenas a dieta de cafeteria, apresentou menor peso relativo do ceco $(0,347 \pm 0,0533)$. Quanto a variável Peso Relativo do Fígado (PRF), não houve diferença estatística entre os grupos experimentais (Tabela 4).
Não ocorreram diferenças estatisticamente significativas entre as variáveis Consumo Alimentar Total (CAT) e Consumo Alimentar Diário (CAD) (p $=0,209)$ como é possível observar na tabela 4 .

Com relação ao Ganho de Peso (GP) entre os grupos experimentais, de forma positiva, os animais do grupo que receberam dieta de cafeteria suplementada com $6 \%$ de YP, apresentaram menor ganho de peso $(p=0,002)$. O GP foi maior no grupo que recebeu apenas a dieta de cafeteria, conforme mostrado na Tabela 4.

Tabela 4 - Resultados do PRC, PRF, CAT, CAD e GP por grupos experimentais (Alfenas, 2017).

\begin{tabular}{|c|c|c|c|c|c|c|}
\hline Variáveis & $\begin{array}{c}\text { CAF }(n=6) \\
X \pm D P\end{array}$ & $\begin{array}{c}\mathrm{CAF}+\mathrm{YP} \\
(\mathrm{n}=6) \mathrm{X} \pm \mathbf{D P}\end{array}$ & $\begin{array}{c}\text { CAF+FOS } \\
(n=6) X \pm \text { DP }\end{array}$ & $\begin{array}{c}\text { CAF+I }(n=6) \\
X \pm D P\end{array}$ & $\begin{array}{c}\text { CAF+FI }(n=6) \\
\text { X } \pm \text { DP }\end{array}$ & $\mathbf{P}^{*}$ \\
\hline PRC (g) & $\begin{array}{c}0,35 \pm \\
0,05^{\mathrm{a}}\end{array}$ & $\begin{array}{c}0,48 \pm \\
0,05^{\mathrm{c}}\end{array}$ & $\begin{array}{c}0,61 \pm \\
0,14^{\mathrm{b}}\end{array}$ & $\begin{array}{c}0,52 \pm \\
0,09^{\mathrm{c}}\end{array}$ & $\begin{array}{c}0,59 \pm \\
0,09^{\mathrm{c}}\end{array}$ & $<0,001$ \\
\hline PRF (g) & $\begin{array}{l}2,75 \pm \\
0,45^{\text {a }}\end{array}$ & $\begin{array}{c}3,22 \pm \\
0,18^{\mathrm{a}}\end{array}$ & $\begin{array}{c}3,11 \pm \\
0,25^{\mathrm{a}}\end{array}$ & $\begin{array}{c}2,82 \pm \\
0,26^{\mathrm{a}}\end{array}$ & $\begin{array}{c}2,91 \pm \\
0,52^{\mathrm{a}}\end{array}$ & 0,164 \\
\hline GP (g) & $\begin{array}{c}142,33 \pm \\
32,00^{\mathrm{a}}\end{array}$ & $\begin{array}{l}74,67 \pm \\
21,19^{\mathrm{b}}\end{array}$ & $\begin{array}{c}117,33 \pm \\
38,42^{\mathrm{a}}\end{array}$ & $\begin{array}{c}128,50 \pm \\
33,63^{\mathrm{a}}\end{array}$ & $\begin{array}{l}80,67 \pm \\
25,53^{\mathrm{b}, \mathrm{c}}\end{array}$ & 0,002 \\
\hline CAT (g) & $\begin{array}{c}560,15 \pm \\
100,68^{\mathrm{a}}\end{array}$ & $\begin{array}{c}506,84 \pm \\
47,21^{\mathrm{a}}\end{array}$ & $\begin{array}{c}576,01 \pm \\
78,92^{\text {a }}\end{array}$ & $\begin{array}{c}532,58 \pm \\
72,92^{\mathrm{a}}\end{array}$ & $\begin{array}{c}485,03 \pm \\
49,53^{\mathrm{a}}\end{array}$ & 0,209 \\
\hline CAD (g) & $\begin{array}{c}20,00 \pm \\
3,59^{\mathrm{a}}\end{array}$ & $\begin{array}{c}18,10 \pm \\
1,68^{\mathrm{a}} \\
\end{array}$ & $\begin{array}{c}20,57 \pm \\
2,81^{\mathrm{a}} \\
\end{array}$ & $\begin{array}{c}19,02 \pm \\
2,59^{\mathrm{a}} \\
\end{array}$ & $\begin{array}{c}17,32 \pm \\
1,76^{\mathrm{a}} \\
\end{array}$ & 0,209 \\
\hline
\end{tabular}

Fonte: Autores.

PRC: Peso Relativo do Ceco; PRF: Peso relativo do fígado; GP: Ganho de Peso; CAT: Consumo Alimentar Total; CAD: Consumo Alimentar Diário; CAF: Grupo controle com dieta de cafeteria; CAF+YP: Grupo dieta de cafeteria suplementada com 6\% de FOS e Inulina do Yacon em pó; CAF+FOS: Grupo dieta de cafeteria suplementada com 6\% de FOS purificada; CAF+I: Grupo dieta de cafeteria suplementada com $6 \%$ de inulina purificada; CAF+FI: Grupo dieta de cafeteria suplementada com 3\% de FOS e 3\% de inulina purificados. X: média, \pm DP: desvio padrão. Valores de média \pm desvio padrão seguidos de pelo menos uma letra minúscula na linha, quando diferentes, representam que há diferença entre os grupos quando comparados pelo teste de ANOVA ( $<<0,05$ ), seguido pelo teste de comparação de médias de Duncan $(\mathrm{p}<0,05)$. Teste de normalidade utilizado: Shapiro-Wilk.Quando letras iguais, indica que não existe diferença entre os grupos utilizando os testes supracitados.

Em relação aos parâmetros bioquímicos, não houve diferença nas variáveis: colesterol total, LDL-c, HDL-c e glicemia. Os parâmetros VLDL e triglicerídeos apresentaram diferenças significativas $(p<0,010)$, com os maiores valores sendo exibidos pelo grupo que recebeu dieta de cafeteria e os menores valores no grupo que recebeu suplementação de FOS e Inulina purificados, conforme abordado na Tabela 5. 
Tabela 5 - Variáveis bioquímicas por grupos experimentais (Alfenas, 2017).

\begin{tabular}{lcccccc}
\hline $\begin{array}{c}\text { Variáveis } \\
(\mathbf{m g} / \mathbf{d L})\end{array}$ & $\begin{array}{c}\mathbf{C A F} \\
(\mathbf{n}=\mathbf{6})\end{array}$ & $\begin{array}{c}\mathbf{C A F}+\mathbf{Y P} \\
(\mathbf{n = 6})\end{array}$ & $\begin{array}{c}\mathbf{C A F}+\mathbf{F O S} \\
(\mathbf{n}=\mathbf{6})\end{array}$ & $\begin{array}{c}\mathbf{C A F + I} \\
(\mathbf{n}=\mathbf{6})\end{array}$ & $\begin{array}{c}\mathbf{C A F + F I} \\
(\mathbf{n}=\mathbf{6})\end{array}$ & $\mathbf{p}^{*}$ \\
\hline Colesterol & $124,95 \pm$ & $133,95 \pm$ & $128,02 \pm$ & $138,85 \pm$ & $140,69 \pm$ & 0,718 \\
$(\mathrm{X} \pm \mathrm{DP})$ & $14,42^{\mathrm{a}}$ & $29,81^{\mathrm{a}}$ & $28,76^{\mathrm{a}}$ & $18,39^{\mathrm{a}}$ & $18,97^{\mathrm{a}}$ & \\
Glicemia & $154,53 \pm$ & $169,37 \pm$ & $167,31 \pm$ & $182,97 \pm$ & $188,74 \pm$ & 0,060 \\
$(\mathrm{X} \pm \mathrm{DP})$ & 19,52 & 30,44 & 20,97 & 29,13 & 15,73 & \\
HDL-c & $59,36 \pm$ & $67,14 \pm$ & $51,84 \pm$ & $71,10 \pm$ & $65,93 \pm$ & 0,420 \\
$(\mathrm{X} \pm \mathrm{DP})$ & $27,33^{\mathrm{a}}$ & $16,93^{\mathrm{a}}$ & $4,89^{\mathrm{a}}$ & $18,8^{\mathrm{a}}$ & $8,16^{\mathrm{a}}$ & \\
Triglicerídeos & $166,49 \pm$ & $128,69 \pm$ & $78,82 \pm$ & $61,53 \pm$ & $41,82 \pm$ & 0,010 \\
$(\mathrm{X} \pm \mathrm{DP})$ & $98,81^{\mathrm{a}}$ & $97,82^{\mathrm{a}}$ & $59,14^{\mathrm{a}}$ & $20,05^{\mathrm{a}}$ & $15,35^{\mathrm{a}, \mathrm{b}}$ & \\
VLDL & $33,297 \pm$ & $25,73 \pm$ & $15,764 \pm$ & $12,306 \pm$ & $8,365 \pm$ & 0,010 \\
$(\mathrm{X} \pm \mathrm{DP})$ & $19,76^{\mathrm{a}}$ & $19,56^{\mathrm{a}, \mathrm{b}}$ & $11,8^{\mathrm{a}, \mathrm{b}}$ & $4,00^{\mathrm{a}, \mathrm{b}}$ & $3,06^{\mathrm{b}}$ & \\
LDL-c & $54,003 \pm$ & $38,808 \pm$ & $57,045 \pm$ & $59,012 \pm$ & $57,914 \pm$ & 0,578 \\
$(\mathrm{X} \pm \mathrm{DP})$ & $35,51^{\mathrm{a}}$ & $27,2^{\mathrm{a}}$ & $38,89^{\mathrm{a}}$ & $12,40^{\mathrm{a}}$ & $24,13^{\mathrm{a}}$ & 0,578 \\
\hline
\end{tabular}

Fonte: Autores.

CAF: Grupo controle com dieta de cafeteria; CAF+YP: Grupo dieta de cafeteria suplementada com 6\% de FOS e Inulina do Yacon em pó; CAF+FOS: Grupo dieta de cafeteria suplementada com 6\% de FOS purificada; CAF+I: Grupo dieta de cafeteria suplementada com $6 \%$ de inulina purificada; CAF+FI: Grupo dieta de cafeteria suplementada com $3 \%$ de FOS e $3 \%$ de inulina purificados. X: média, \pm DP: desvio padrão. Md.: Mediana (mínimo e máximo). Valores de média \pm desvio padrão seguidos de pelo menos uma letra minúscula na linha, quando diferentes, representam que há diferença entre os grupos quando comparados pelo teste ANOVA $(\mathrm{p}<0,05)$. Quando seguidos de letras minúsculas diferentes na linha, diferem entre si pelo teste de Kruskal -Wallis seguido pelo teste de comparação de média de Dunn's ou *Duncan $(\mathrm{P}<0,05)$. Teste de normalidade utilizado: Shapiro-Wilk.Quando letras iguais, indica que não existe diferença entre os grupos utilizando os testes supracitados.

Quanto aos tecidos adiposos subcutâneo e epididimal, foi possível verificar diferenças significantes entre os grupos, sendo os menores valores apresentados pelo grupo FOS + Inulina, conforme Tabela 6.

Tabela 6 - Tecidos adiposos por grupos experimentais (Alfenas, 2017).

\begin{tabular}{ccccccc}
\hline Variáveis & $\begin{array}{c}\mathbf{C A F} \\
(\mathbf{n}=\mathbf{6})\end{array}$ & $\begin{array}{c}\mathbf{C A F}+\mathbf{Y P} \\
(\mathbf{n}=\mathbf{6})\end{array}$ & $\begin{array}{c}\mathbf{C A F}+\mathbf{F O S} \\
(\mathbf{n}=\mathbf{6})\end{array}$ & $\begin{array}{c}\mathbf{C A F}+\mathbf{I} \\
(\mathbf{n}=\mathbf{6})\end{array}$ & $\begin{array}{c}\mathbf{C A F}+\mathbf{F I} \\
(\mathbf{n}=\mathbf{6})\end{array}$ & $\mathbf{p}^{*}$ \\
\hline $\begin{array}{c}\text { TA Epididimal } \\
(\mathrm{X} \pm \mathrm{DP})\end{array}$ & $2,32 \pm 0,289^{\mathrm{a}}$ & $1,54 \pm 0,143^{\mathrm{d}, \mathrm{b}}$ & $1,36 \pm 0,19^{\mathrm{c}, \mathrm{b}}$ & $1,68 \pm 0,26^{\mathrm{d}, \mathrm{b}}$ & $1,13 \pm 0,13^{\mathrm{b}}$ & 0,008 \\
$\begin{array}{c}\text { TA Mesentérico } \\
(\mathrm{X} \pm \mathrm{DP})\end{array}$ & $5,48 \pm 0,84^{\mathrm{a}}$ & $3,52 \pm 0,49^{\mathrm{a}}$ & $4,23 \pm 0,53^{\mathrm{a}}$ & $4,54 \pm 0,46^{\mathrm{a}}$ & $3,42 \pm 0,38^{\mathrm{a}}$ & 0,095 \\
$\begin{array}{c}\text { TA Subcutâneo } \\
\quad(\mathrm{X} \pm \text { DP) }\end{array}$ & $6,05 \pm 2,61^{\mathrm{a}}$ & $2,57 \pm 0,94^{\mathrm{c}, \mathrm{b}}$ & $4,43 \pm 0,88^{\mathrm{a}, \mathrm{b}}$ & $4,21 \pm 1,88^{\mathrm{a}, \mathrm{b}}$ & $2,42 \pm 0,7^{\mathrm{b}}$ & 0,003 \\
$\begin{array}{c}\text { TA Retroperitoneal } \\
(\mathrm{X} \pm \mathrm{DP})\end{array}$ & $7,35 \pm 4,32^{\mathrm{a}}$ & $3,37 \pm 1,18^{\mathrm{a}}$ & $4,44 \pm 1,53^{\mathrm{a}}$ & $5,93 \pm 2,36^{\mathrm{a}}$ & $2,80 \pm 1,42^{\mathrm{a}}$ & 0,074 \\
\hline
\end{tabular}

Fonte: Autores.

TA: tecido adiposo; CAF: Grupo controle com dieta de cafeteria; CAF+YP: Grupo dieta de cafeteria suplementada com 6\% de FOS e Inulina do Yacon em pó; CAF+FOS: Grupo dieta de cafeteria suplementada com 6\% de FOS purificada; CAF+I: Grupo dieta de cafeteria suplementada com 6\% de inulina purificada; CAF+FI: Grupo dieta de cafeteria suplementada com 3\% de FOS e 3\% de inulina purificados. X: média, \pm DP: desvio padrão. Md.: Mediana (mínimo e máximo). Valores de média \pm desvio padrão seguidos de pelo menos uma letra minúscula na linha, quando diferentes, representam que há diferença entre os grupos, quando comparados pelo teste ANOVA $(\mathrm{p}<0,05)$. Quando seguidos de letras minúsculas diferentes na linha, diferem entre si pelo teste de Kruskal Wallis seguido pelo teste de comparação de média de Dunn's ou *Duncan $(\mathrm{P}<0,05)$. Teste de normalidade utilizado: Shapiro-Wilk. representam que há diferença quando comparados pelo teste. 


\section{Discussão}

No presente estudo a variável peso relativo do ceco apresentou diferenças significativas $(\mathrm{p}<0,001)$. O aumento cecal pode ocorrer devido ao efeito osmótico que estes prebióticos exercem, retendo água no tecido. Além disso, também pode associar-se à produção de ácidos graxos de cadeia curta (AGCC): acetato, butirato e propionato, os quais conseguem estimular a hipertrofia do ceco, por meio da atividade metabólica, ${ }^{(19)}$ caracterizada pela proliferação celular estimulada pelos AGCC e pela ação das bifidobactérias. ${ }^{(20)}$ Entretanto, o uso de FOS e inulina não deve ser indiscriminado, uma vez que podem causar desconfortos intestinais, como gases e diarreia.

A variável peso do fígado não apresentou diferença estatística. Dessa forma, em seu estudo Borba et al. ${ }^{(21)}$ relataram o aumento do peso corporal de ratos submetidos à dieta hiperlipídica, porém, assim como no presente estudo, também não foram apresentadas diferenças no peso do fígado, o que pode ser justificado pelo fato de que não apenas a densidade energética da dieta está relacionada com as alterações metabólicas, mas possivelmente a proporção dos nutrientes oferecidos também serem determinantes para modificações fisiológicas.

O ganho de peso foi maior no grupo que não recebia suplementação de prebióticos. Esse fato pode acontecer pela menor presença de fibras a qual este grupo foi submetido. As fibras solúveis, como FOS e inulina, apresentam a propriedade de formar géis no intestino delgado, quando em contanto com a água. Este gel reduz o contato dos nutrientes com a mucosa, restringindo a absorção destes e consequentemente o total energético. ${ }^{(22)}$ Após serem fermentados, os prebióticos dão origem aos AGCC, estes apresentam a propriedade de proteger contra a obesidade induzida sem causar hipofagia, ao mesmo tempo, o butirato tem a capacidade de aumentar o gasto energético, reduzindo o ganho de peso ${ }^{(23)}$. Além disso, AGCC ligam-se à receptores Gpr43 expressos em diversos tecidos, incluindo o cólon, estimulando a secreção de glucagon-like peptide 1 (GLP-1), um hormônio anorexígeno que melhora a sensibilidade à insulina e através da supressão desta, previne o acúmulo de tecido adiposo.(1) Os AGCC podem também interagir com o receptor Gpr41, situado nas células enteroendócrinas, liberando o hormônio PYY, que apresenta a capacidade de diminuir a extração de energia dos componentes alimentares, além de reduzir a motilidade intestinal, reduzindo, consequentemente, o trânsito intestinal. (24)

Ainda que o ganho de peso tenha sido maior entre os animais que receberam apenas dieta de cafeteria, não foram apresentadas diferenças estatísticas quanto ao consumo total e diário de dieta entre os grupos experimentais. ${ }^{(24)}$ Uma possível explicação para o consumo semelhante entre os grupos experimentais seria o fato de que a dieta de cafeteria ser composta por alimentos palatáveis de alta densidade energética estimulando a sensação de prazer. Sendo assim, os animais acabam por apresentar hiperfagia. ${ }^{(25,26)}$

Nesse sentido, South et al. ${ }^{(27)}$ mostraram que a exposição a dietas palatáveis tornava os ratos hiperfágicos, enquanto que ao serem expostos à ração padrão, os animais apresentavam baixo consumo alimentar e consequente perda de peso.

Quanto ao colesterol total, também não foram observadas alterações estatísticas significativas. A não variação do colesterol possivelmente pode ser explicada tomando como base a desregulação da proteína 4 semelhante à angiopoietina (ANGPTL4). Os AGCC como propionato e acetato produzidos pelos prebióticos estão intimamente envolvidos na regulação da síntese do colesterol hepático e acredita-se que o aumento no consumo de prebióticos poderia aumentar o influxo de AGCC no fígado. (28) $\mathrm{O}$ aumento destes ácidos graxos poderia então gerar uma desregulação na produção de ANGPTL4, aumentando sua produção. Ainda neste sentido, estudos apontam que a ANGPTL4 é responsável pela inibição da Lipoproteína Lipase (LPL), sendo que, 
esta última tem como função a depuração lipídica. Sendo assim, o acetato e o propionato, conhecidos por serem AGCC, aumentariam a produção da ANGPTL4 e, por consequência, haveria a inibição da LPL, não havendo assim a depuração lipídica, o que manteria o nível do colesterol total aumentado ou mesmo estacionado, como no presente estudo. $(29,30)$

Em relação ao nível glicêmico entre os grupos comparados, deve-se considerar que não houve aferição da glicemia antes do fornecimento da dieta de cafeteria, impossibilitando o conhecimento quanto à homogeneidade glicêmica dos animais em estudo. Para Flesch, Poziomych e Damin ${ }^{(31)}$ os efeitos da utilização da inulina e FOS no perfil glicêmico não estão completamente elucidados, uma vez que são contraditórios e dependem de condições como jejum ou pós-prandial além do diagnóstico de diabetes.

Os valores de HDL não apresentaram nenhuma diferença significativa entre os grupos experimentais. Sendo assim, sugere-se que seria necessário um tempo de adaptação metabólica dos animais. A literatura recomenda que todo experimento seja feito em um intervalo de 8 semanas, afim de que se possa observar alterações nos parâmetros avaliados. ${ }^{(32)}$

Mesmo com o fornecimento de uma dieta com elevado teor lipídico notou-se redução de VLDL e triglicerídeos entre os grupos estudados. Em estudos utilizando a inulina, verificou-se que a diminuição das concentrações séricas de VLDL, seja resultante do decréscimo da síntese hepática de triglicerídeos e catabolismo das lipoproteínas que o contém. Sugere-se que a hipotrigliceridemia seja decorrente da redução da atividade hipogênica no fígado, ou seja, menor reesterificação hepática de ácidos graxos, com a consequente diminuição de triglicerídeos na circulação. ${ }^{(19)}$ Outro estudo realizado com o mesmo prebiótico mostrou eficácia na redução de triglicerídeos e lipogênese hepática com a utilização de $10 \mathrm{~g} /$ dia de inulina por seis semanas. ${ }^{(33)}$
Além disso, observam-se os efeitos dos frutanos na modulação da adipogênese, uma vez que animais alimentados com FOS e Inulina apresentaram deposição de tecido adiposo epididimal e subcutâneo inferior aos demais grupos analisados. É possível que estes animais suplementados com FOS e Inulina tenham tido um aumento da lipólise, o que justifica a perda de peso desses animais, ainda que o consumo fosse semelhante. ${ }^{(24)}$ Em seu experimento Dewulf e colaboradores, ${ }^{(34)}$ observaram que camundongos suplementados com frutanos tipo inulina tinham um aumento expressivo da lipólise no tecido subcutâneo, o que explica os valores significativos de redução deste tecido adiposo nos grupos FOS e Inulina e Yacon. Além disso, nesse mesmo estudo, ${ }^{(34)}$ perceberam a ativação de receptores que são ativados por proliferadores de peroxissoma gama (PPAR $\gamma$ ) acabou diminuindo a expressão de proteínas ligadoras de ácidos graxos. Tais proteínas participam de processos de captação e diferenciação de adipócitos, sendo assim, estaria diminuída a lipogênese.

No presente estudo, a gordura epididimal mostrou-se significativa entre os grupos comparados. Considera-se tecido adiposo visceral a associação da gordura epididimal, retroperitoneal e mesentérica. ${ }^{(35)}$ Quando comparado à gordura subcutânea, o tecido visceral é considerado mais ativo, ou seja, mais sensível à lipólise por meio das catecolaminas e $\beta$-adrenorreceptores. Além disso, é considerado mais resistentes a ação da insulina, liberando quantidades significativas de ácidos graxos livres. Trata-se também de um tecido com secreções significativas de adipocinas próinflamatórias, como resistina, angiotensina I, PAI-1, PCR, IL-6. ${ }^{(36)}$

Diversos autores defendem a relação da gordura visceral com resistência à insulina e risco cardiovascular, além de doença arterial coronariana mesmo em indivíduos não obesos. ${ }^{(37)}$ 


\section{Conclusão}

Por meio deste trabalho verificou-se a influência dos prebióticos FOS e Inulina nos seguintes parâmetros: peso relativo do ceco, ganho de peso, VLDL, triglicerídeos, tecidos adiposos subcutâneo e epididimal, mostrando efeitos sistêmicos benéficos.

As demais variáveis analisadas foram divergentes com os dados da literatura. Acredita-se que o tempo de intervenção não tenha sido suficiente para induzir algumas respostas metabólicas. Sendo assim, novos estudos associados aos frutanos tipo inulina e Dieta de Cafeteria precisam ser realizados.

\section{Agradecimentos}

Agradecemos o laboratório de Nutrição Experimental da Unifal-MG pela disponibilidade, assim como, ao Centro de Raízes e Amidos Tropicais (CERAT - Botucatu) através das análises bromatológicas e ao Programa Institucional de Iniciação Científica Voluntária (PIVIC) pela oportunidade de expansão dos conhecimentos científicos.

\section{Referências}

1 Moraes ACF, Fernandes GR, da Silva IT, AlmeidaPititto B, Gomes EP, da Costa PereiraA, etal. Enterotype may drive the dietary-associated cardiometabolic risk factors. frontiers cell Infect Microbiol. 2017 Feb 23;7:47. doi: 10.3389/fcimb.2018.00249

2 Rial AR, Karelis AD, Bergeron KF, Mounier C. Gut microbiota and metabolic health: the potential beneficial effects of a medium chain triglyceride diet in obese individuals. Nutrients. 2016 May 12;8(5). pii: E281. doi: 10.3390/nu8050281

3 Steppa R, Szkudelska K, Wójtowski J, Stanisz M, Szumacher-Strabel M, Czyzak-Runowska G, et al. The metabolic profile of growing lambs fed diets rich in unsaturated fatty acids. J Anim Physiol Anim Nutr (Berl). 2014 Oct;98(5):914-20. doi: 10.1111/ jpn. 12158

4 Pons Z, Margalef M, Bravo FI, Arola-Arnal A, Muguerza B. Chronic administration of grapeseed polyphenols attenuates the development of hypertension and improves other cardiometabolic risk factors associated with the metabolic syndrome in cafeteria diet-fed rats. Br J Nutr. 2017 Jan;117(2):200208. doi: 10.1017/S0007114516004426

5 Silva ACC, Silva NA, Pereira MCS, Vassimon HS. Alimentos contendo ingredientes funcionais em sua formulação: revisão de artigos publicados em revistas brasileiras. Revista Conexão Ciência. 2016 12;11(2):133-44.

6 Conrado BA, de Souza SA, Mallet ACT, de Souza EB, dos Santos Neves A, Saro MLG. Disbiose intestinal em idosos e aplicabilidade dos probióticos e prebióticos. Cadernos UniFOA. 2018 04;13(6):71-78.

7 Tufarelli V, Laudadio V. An overview on the functional food concept: prospectives and applied researches in probiotics, prebiotics and synbiotics. JEBA: Journal of Experimental Biology and Agricultural Sciences. 2016 6;4(3):273-78. doi: http://dx.doi. org/10.18006/2016.4(3S).273.278

8 Uyeda M, Buonom HCD, Gonzaga MFN, de Carvalho FLO. Probióticos e prebióticos: benefícios acerca da literatura. Revista de Saúde UniAGES. 2017 2;1(1):58-87.

9 Santos LC, Cançado IAC. Probióticos e prebióticos: vale a pena incluí-los em nossa alimentação! Revista Digital FAPAM. 2009 10;1(1):308-17.

10 Javier Fernández, Redondo-Blanco S, Gutiérrezdel-Río I, Miguélez EM, Villar CJ, Lombó F. Colon microbiota fermentation of dietary prebiotics towards short-chain fatty acids and their roles as anti-inflammatory and antitumour agents: a review. J Funct Foods. 2016;8(25):511-22. doi: 10.1016/j. jff.2016.06.032

11 Silva Sacramento M, Silva PSRC, Tavares MIB. Batata yacon-alimento funcional. Revista Semioses. 2017;11(3):43-8.

12 Caetano BFR, Moura NA, Almeida APS, Dias MC, Barbisan LF. Yacon (Smallanthus sonchifolius) as a food supplement: health-promoting benefits of fructooligosaccharides. Nutrients. 2016 Jul 21;8(7). pii: E436. doi: 10.3390/nu8070436

13 Association of Official Analytical Chemists.Official methods of analysis. $9^{\text {th ed }}$. Local: AOAC; 2005.

14 Kaneko T, Kudo, T, Horikoshi K. Comparacion of CD composiotion produced by chimeric CGTases. Agric Biol Chem. 1990;1(54):197-201.

15 Costa NMB, Peluzio MCG, Martino HSD, Henriques GS. Nutrição experimental: teoria e prática. 1st ed. Rubio; 2014. 
16 Campión J, Martínez JA. Ketoconazole, an antifungal agent, protects against adiposity induced by a cafeteria diet. Horm Metab Res. 2004 Jul;36(7):48591. doi: $10.1055 / \mathrm{s}-2004-825729$

17 Friedewald WT, Levy R, Frederickson DS. Estimation of the concentration of low-density lipoprotein cholesterol in plasma, without use of the preparative ultracentrifuge. Clini Chem. 1972;46(9):1351-6.

18 Sposito AC., Caramelli B, Fonseca FAH., Bertolami MC., Afiune Neto A, Souza AD et al. IV diretriz brasileira sobre dislipidemias e prevenção da aterosclerose: Departamento de Aterosclerose da Sociedade Brasileira de Cardiologia. Arq. Bras. Cardiol. 2007 Apr; 88(Suppl 1):2-19.

19 Martins JFL, Rafael VC, Filomena EA, Rodrigues FC, Sartori SSR, Ferreira CLLF. Produto a base de yacon e modulação da microbiota, perfil de ácidos graxo e lipídico em ratas ovariectomizadas. Rev. Nutr. [Internet]. 2016 Oct [cited 2018 Aug 07]; 29(5):709-719. Disponível em: http://www. scielo.br/scielo.php?script $=$ sci_arttext\&pid=S1415$52732016000500709 \& \operatorname{lng}=\mathrm{en}$. org/10.1590/1678-98652016000500009

20 Lima GC, Maróstica Junior MR. Efeito dos oligossacarídeos FOS e GOS na microbiota intestinal e no $\mathrm{pH}$ do conteúdo cecal de ratas Wistar em desenvolvimento [Faculdade de Engenharia de Alimentos Departamento de Alimentos e Nutrição]. Unicamp; 2010.

21 Borba AJ, Rocha MGM, da Silva MF, Tibúrcio DTS, de Lima Pereira SA, Reis LC, et al. Dieta hiperlipídico-proteica utilizada para emagrecimento induz obesidade em ratos. Rev. Nutr. [Internet]. 2011; 24(4):519-528. Disponível em: http://www. scielo.br/scielo.php?script=sci_arttext\&pid=S141552732011000400001\&lng=en. $\quad \mathrm{http}: / / \mathrm{dx}$. doi. org/10.1590/S1415-52732011000400001

22 Lage FB, Guerra HMM, Pelogia NCC. Efeito da farinha da casca de maracujá no peso e no índice glicêmico de ratos. RBNE: Revista Brasileira de Nutrição Esportiva. 2014 3;8(44):84-91.

23 Chakraborti CK. New-found link between microbiota and obesity. World J Gastrointest Pathophysiol. 2015 11;6(4):110-119. Disponível em: https://www. ncbi.nlm.nih.gov/pmc/articles/PMC4644874/. doi: 10.4291/wjgp.v6.i4.110

24 Frota KMG, Soares NRM, Muniz VRC, Fontenelle LC, Carvalho CMRG. Efeito de prebióticos e probióticos na microbiota intestinal e nas alterações metabólicas de indivíduos obesos. Nutrire. 2015
8;40(2):173-87. http://dx.doi.org/10.4322/23167874.55314

25 Goularte JF, Ferreira MBC, Sanvitto GL. Effects of food pattern change and physical exercise on cafeteria diet-induced obesity in female rats. $\mathrm{Br} \mathrm{J}$ Nutr. 2012 Oct 28;108(8):1511-8. doi: 10.1017/ S0007114511006933

26 Sampey BP, Vanhoose AM, Winfield HM, Freemerman AJ, Muehlbauer MJ, Fueger PT, et al. Cafeteria diet is a robust model of human metabolic syndrome with liver and adipose inflammation: comparison to highfat diet. Obesity. 2011 6;19(6):1109-17. doi: 10.1038/ oby. 2011.18

27 South T, Westbrook F, Morris MJ. Neurological and stress related effects of shifting obese rats from a palatable diet to chow and lean rats from chow to a palatable diet. Physiol Behav. 2012 2;105(4):105257. doi: 10.1016/j.physbeh.2011.11.019

28 He M, Shi B. Gut microbiota as a potential target of metabolic syndrome: the role of probiotics and prebiotics. Cell Biosci. 2017;7(1):54. doi: 10.1186/ s13578-017-0183-1

29 Dijk W, Kersten S. Regulation of lipoprotein lipase by Angptl4. Trends Endocrinol Metab. 2014 Mar;25(3):146-55. doi: 10.1016/j.tem.2013.12.005

30 Kim YA, Keogh JB, Clifton PM. Probiotics, prebiotics, synbiotics and insulin sensitivity. Nutr Res Rev. 2017 Jun;31(1):35-51. doi: 10.1017/S095442241700018X

31 Flesch AGT, Poziomyck AK, Damin DDC. The therapeutic use of symbiotics. Arq Bras Cir Dig. 2014;27(3):206-9. Disponível em: https://www. ncbi.nlm.nih.gov/pmc/articles/PMC4676381/. doi: 10.1590/S0102-67202014000300012

32 Marques ADCR, Gabbiatti GC, Gravena AAF, Amaral VD. Influência das dietas hipercalóricas sobre os parâmetros de obesidade, dislipidemia e hiperglicemia em ratos. Saúde e Pesquisa. 2015;8(1):55-62. doi: 10.17765/1983-1870.2015v8n $1 \mathrm{p} 55-62$

33 Huang W, Lin C, Hsu Y, Chiu Y, Chen Y, Wu $\mathrm{M}$, et al. Inulin and fibersol-2 combined have hypolipidemic effects on high cholesterol dietinduced hyperlipidemia in hamsters. Molecules (Basel, Switzerland). 2016;3(21):313. doi: 10.3390/ molecules21030313

34 Dewulf EM, Cani PD, Neyrinck AM, Possemiers $\mathrm{S}$, Holle AV, Muccioli GG, et al. Inulin-type fructans with prebiotic properties counteract GPR43 overexpression and PPAR-related adipogenesis in the 
white adipose tissue of high-fat diet-fed mice. J Nutr Biochem. 2011 Aug;22(8):712-22. doi: 10.1016/j. jnutbio.2010.05.009

35 Frankenfeld SP, Casimiro-Lopes G, Boaventura G, Conceição EPSD, Oliveira E, Moura E, et al. Efeitos do treinamento em esteira em ratos adultos obesos programados pela superalimentação pós-natal. Arquivos em Movimento. 2016;12(1):65-90.

36 Hermsdorff HHM, Monteiro JBR. Gordura visceral, subcutânea ou intramuscular: onde está o problema? Arq Bras Endocrinol Metab [Internet]. 2004 Dec;
48(6):803-811. Disponível em: http://www.scielo. $\mathrm{br} / \mathrm{scielo}$.php? script $=$ sci_arttext\&pid $=\mathrm{S} 0004$ 27302004000600005\&lng=en. $\quad$ http://dx.doi. org/10.1590/S0004-27302004000600005

37 Ribeiro Filho FF, Mariosa LS, Ferreira SRG, Zanella MT. Gordura visceral e síndrome metabólica: mais que uma simples associação. Arq Bras Endocrinol Metab [Internet]. 2006 Apr; 50(2):230-238. Available from: http://www.scielo. $\mathrm{br} / \mathrm{scielo} . \mathrm{php}$ ?script $=$ sci_arttext\&pid $=\mathrm{S} 0004-$ $27302006000200009 \& \operatorname{lng}=$ en. http://dx.doi. org/10.1590/S0004-27302006000200009 